\title{
Longitudinal Population Dynamics of Staphylococcus aureus in the Nasopharynx During the First Year of Life
}

\begin{abstract}
Shima M. Abdulgader ${ }^{1 *}$, Lourens Robberts ${ }^{1}$, Jordache Ramjith ${ }^{2}$, Polite M. Nduru ${ }^{3}$, Felix Dube ${ }^{1,4}$, Sugnet Gardner-Lubbe ${ }^{5}$, Heather J. Zar ${ }^{3}$ and Mark P. Nicol1,6,7

${ }^{1}$ Division of Medical Microbiology, Department of Pathology, Faculty of Health Sciences, University of Cape Town, Cape Town, South Africa, ${ }^{2}$ Division of Epidemiology and Biostatistics, School of Public Health and Family Medicine, University of Cape Town, Cape Town, South Africa, ${ }^{3}$ Department of Paediatrics and Child Health, Red Cross War Memorial Children's Hospital and SA-MRC Unit on Child and Adolescent Health, University of Cape Town, Cape Town, South Africa, ${ }^{4}$ Department of Molecular and Cell Biology, Faculty of Science, University of Cape Town, Cape Town, South Africa, ${ }^{5}$ Department of Statistics and Actuarial Science, Stellenbosch University, Stellenbosch, South Africa, ${ }^{6}$ Institute of Infectious Disease \& Molecular Medicine, Faculty of Health Sciences, University of Cape Town, Cape Town, South Africa, ${ }^{7}$ School of Biomedical Sciences, University of Western Australia, Perth, WA, Australia
\end{abstract}

OPEN ACCESS

Edited by:

Yasir Muhammad, King Abdulaziz University,

Saudi Arabia

Reviewed by:

Markus Hilty,

University of Bern, Switzerland

Imran Khan,

Macau University of Science and Technology, Macau

*Correspondence:

Shima M. Abdulgader shimamohd1@gmail.com

Specialty section: This article was submitted to

Evolutionary and Genomic Microbiology

a section of the journal

Frontiers in Genetics

Received: 04 December 2018 Accepted: 25 February 2019 Published: 15 March 2019

Citation: Abdulgader SM, Robberts $L$, Ramjith J, Nduru PM, Dube F, Gardner-Lubbe S, Zar HJ and Nicol MP (2019) Longitudinal Population Dynamics

of Staphylococcus aureus in the Nasopharynx During the First Year of Life. Front. Genet. 10:198. doi: 10.3389/fgene.2019.00198
Background: Staphylococcus aureus colonization is a risk factor for invasive disease. Few studies have used strain genotype data to study $S$. aureus acquisition and carriage patterns. We investigated $S$. aureus nasopharyngeal carriage in infants in an intensively sampled South African birth cohort.

Methods: Nasopharyngeal swabs were collected at birth and fortnightly from 137 infants through their first year of life. S. aureus was characterized by spa-typing. The incidence of $S$. aureus acquisition, and median carriage duration for each genotype was determined. S. aureus carriage patterns were defined by combining the carrier index (proportion of samples testing positive for $S$. aureus) with genotype diversity measures. Persistent or prolonged carriage were defined by a carrier index $\geq 0.8$ or $\geq 0.5$, respectively. Risk factors for time to acquisition of $S$. aureus were determined.

Results: Eighty eight percent (121/137) of infants acquired S. aureus at least once. The incidence of acquisition at the species and genotype level was 1.83 and 2.8 episodes per child-year, respectively. No children had persistent carriage (defined as carrier index of $>0.8$ ). At the species level $6 \%$ had prolonged carriage, while only $2 \%$ had prolonged carriage with the same genotype. Carrier index correlated with the absolute number of spa-CCs carried by each infant $(r=0.5 ; 95 \% \mathrm{Cl} 0.35-0.62)$. Time to first acquisition of S. aureus was shorter in children from households with $\geq 5$ individuals (HR $1.06,95 \% \mathrm{Cl}$ 1.07-1.43), with S. aureus carrier mothers (HR; $1.5,95 \% \mathrm{Cl} 1.2-2.47)$, or with a positive tuberculin skin test during the first year of life (HR; 1.81, 95\% Cl 0.97-3.3).

Conclusion: Using measures of genotype diversity, we showed that $S$. aureus NP carriage is highly dynamic in infants. Prolonged carriage with a single strain occurred rarely; persistent carriage was not observed. A correlation was observed between carrier index and genotype diversity.

Keywords: Staphylococcus aureus, carriage, infancy, genotype diversity, longitudinal 


\section{INTRODUCTION}

Staphylococcus aureus colonizes the mucosal and epithelial surfaces of several body sites such as the nose, throat, gut, axilla, groin, vagina, and perineum. However, the nasal cavity is considered the primary anatomical site for colonization (Verhoeven et al., 2014). Asymptomatic carriage is a risk factor for infections, both community-acquired (particularly skin and soft tissue infections) and nosocomial (including surgical site infection, catheter-related infections, pneumonia, and endocarditis) (Sakr et al., 2018). Impairment in the local or systemic immune response, for example due to disruption of the skin barrier, extremes of age, or HIV infection are associated with progression to disease (Wertheim et al., 2005; Johannessen et al., 2012). Metabolic adaptation during lack of nutrients and enhanced expression of virulence genes which are mediated by two-component systems and global regulators play critical role in the transition of $S$. aureus from the colonizer to the invasive state (Pohl et al., 2009; Waters et al., 2016).

In adults, longitudinal studies describe three nasal carriage patterns based on the carrier index (the proportion of samples collected from each individual which are positive for $S$. aureus). Individuals with indices $>0.8$ or 1 are considered persistent carriers, individuals with an index of zero are non-carriers, and individuals with indices between 0 and 0.8 are considered intermittent carriers (Van Belkum et al., 1997; Vandenbergh et al., 1999; Blumental et al., 2013; Muthukrishnan et al., 2013; Verhoeven et al., 2014). Approximately one third of adults are considered to be persistent carriers (Wertheim et al., 2005). However, definitions of carriage are influenced by the frequency of sampling, the number of swabs collected and follow up period, which vary between studies (Van Belkum et al., 2009; Muthukrishnan et al., 2013; Verhoeven et al., 2014); seven swabs collected prospectively over 5 weeks were shown to distinguish $S$. aureus non-carriers from intermittent carriers (Nouwen et al., 2004).

Studies of $S$. aureus carriage in healthy populations reported higher persistent carriage rates among children compared to adults (Cunliffe, 1949; Noble et al., 1967; Armstrong-Esther and Smith, 1976). However, longitudinal studies of S. aureus nasal carriage patterns at the species level provide an incomplete description of the true nature of carriage as they are unable to distinguish between persistent carriage with a single strain and sequential carriage with different strains. True persistent colonization would imply carriage of a single genotype over an extended period (Peacock et al., 2003; Lebon et al., 2008). Few studies have used strain genotyping to support their definitions of persistent vs. intermittent nasal carriage, especially among infants (Blumental et al., 2013; Muthukrishnan et al., 2013; Schaumburg et al., 2013). Studies in children that have used strain genotyping, have only collected a very limited number of swabs over the period of study, and are therefore unable to accurately address this question (Blumental et al., 2013; Schaumburg et al., 2013).

In order to address this gap in knowledge, we therefore conducted intensive longitudinal NP sampling of a cohort of infants for 12 months and genotyped S. aureus strains using spa-typing. We determined, for the first time, $S$. aureus NP carriage dynamics (the incidence of $S$. aureus acquisition and number of carriage episodes) both at the species and the genotype levels. We show that whilst acquisition is common, persistent or prolonged carriage with the same genotype is rare in infants.

\section{MATERIALS AND METHODS}

\section{Study Population and Characterization of $S$. aureus}

This study was nested within the Drakenstein Child Health Study - a population based cohort study of a 1143 motherinfant pairs based in a peri-urban community in Cape Town, South Africa (Zar et al., 2014). A total of 885 mother-infant

TABLE 1 | Participant characteristics.

\begin{tabular}{|c|c|}
\hline Characteristics $(n=137)$ & No. (\%) \\
\hline \multicolumn{2}{|l|}{ Gender } \\
\hline Female & $77(56.2)$ \\
\hline Gestational age: median (range; IQR) & $39(27-42 ; 38-39)$ \\
\hline Premature birth ( $<37$ weeks) & $21(15)$ \\
\hline \multicolumn{2}{|l|}{ Mode of delivery } \\
\hline Vaginal & 109(80) \\
\hline Cesarean & 28(20) \\
\hline \multicolumn{2}{|l|}{$\begin{array}{l}\text { Baby feeding method during first } \\
6 \text { months }\end{array}$} \\
\hline Exclusive breast feeding & $38(28)$ \\
\hline Exclusive formula feeding & $60(44)$ \\
\hline Mixed feeding & 39(28) \\
\hline Day care attendance & $81(59)$ \\
\hline HIV exposure & $33(24)$ \\
\hline \multicolumn{2}{|l|}{ Smoke exposure } \\
\hline Smoker father & $84(61)$ \\
\hline Smoker mother & $45(34)$ \\
\hline Both parents & $25(34)$ \\
\hline Either parent smoker & 95(69) \\
\hline Any smoker exposure & 114(83) \\
\hline \multicolumn{2}{|l|}{ Socioeconomic status } \\
\hline Low & $74(54)$ \\
\hline Moderate-high & $63(45)$ \\
\hline Animals in the house & $62(45)$ \\
\hline \multicolumn{2}{|l|}{ Maternal education } \\
\hline Primary & 13(10) \\
\hline Secondary & 115(86) \\
\hline Tertiary & $6(4)$ \\
\hline Infants treated for TB & 18(13) \\
\hline Household size $>5$ & $50(36)$ \\
\hline Number of people in household & $5(1-20 ; 3-6)$ \\
\hline \multicolumn{2}{|l|}{ Median (range; IQR) } \\
\hline Having a sibling younger than 5 yrs & $47(34)$ \\
\hline
\end{tabular}


pairs chose to enroll in an intensive cohort who were followed up fortnightly for the first year of life. Mothers were enrolled during the second trimester of pregnancy; details of the broader study are described elsewhere (Zar et al., 2014). Socioeconomic measures were from a composite validated score, HIV exposure was defined as HIV uninfected infants born to HIV-infected mothers, maternal, or paternal smoking was assessed by selfreport. Tuberculin skin test (TST) reactivity was defined as $\geq 10 \mathrm{~mm}$ in HIV-uninfected children and $\geq 5 \mathrm{~mm}$ in HIVinfected children.

NP swabs (Copan flocked swab, FLOQSwabs ${ }^{\text {TM }}$, COPAN Diagnostics, Murrieta, CA, United States) were collected at birth from mother-infant pairs and fortnightly from infants through their first year of life. One hundred and thirtyseven mother-infant pairs were selected for this study - these were selected as the first infants to complete their first year of life, with a minimum set of $18 \mathrm{NP}$ samples collected from the fortnightly collection. Clinical and demographical data were collected at routine study visits. Swabs were inoculated onto Mannitol Salt Agar (MSA) (National Health Laboratory Services, Green Point Media Laboratory Cape Town, South Africa) and incubated at $35^{\circ} \mathrm{C}$ for $18-24 \mathrm{~h}$ in room air. Mannitol-positive and DNase-positive isolates were identified as S. aureus (Kateete et al., 2010). S. aureus isolates were characterized by spa-typing and clustered based on their genetic relatedness into spa-clonal complexes (CCs) using the Based Upon Repeat Pattern (BURP) clustering algorithm of the Ridom Staph Type software (Ridom GmbH, Münster, Germany).

This study was approved by the Human Research Ethics Committee of the Faculty of Health Sciences, University of Cape Town (HREC ref: 401/2009 and 740/2013) and the Western Cape

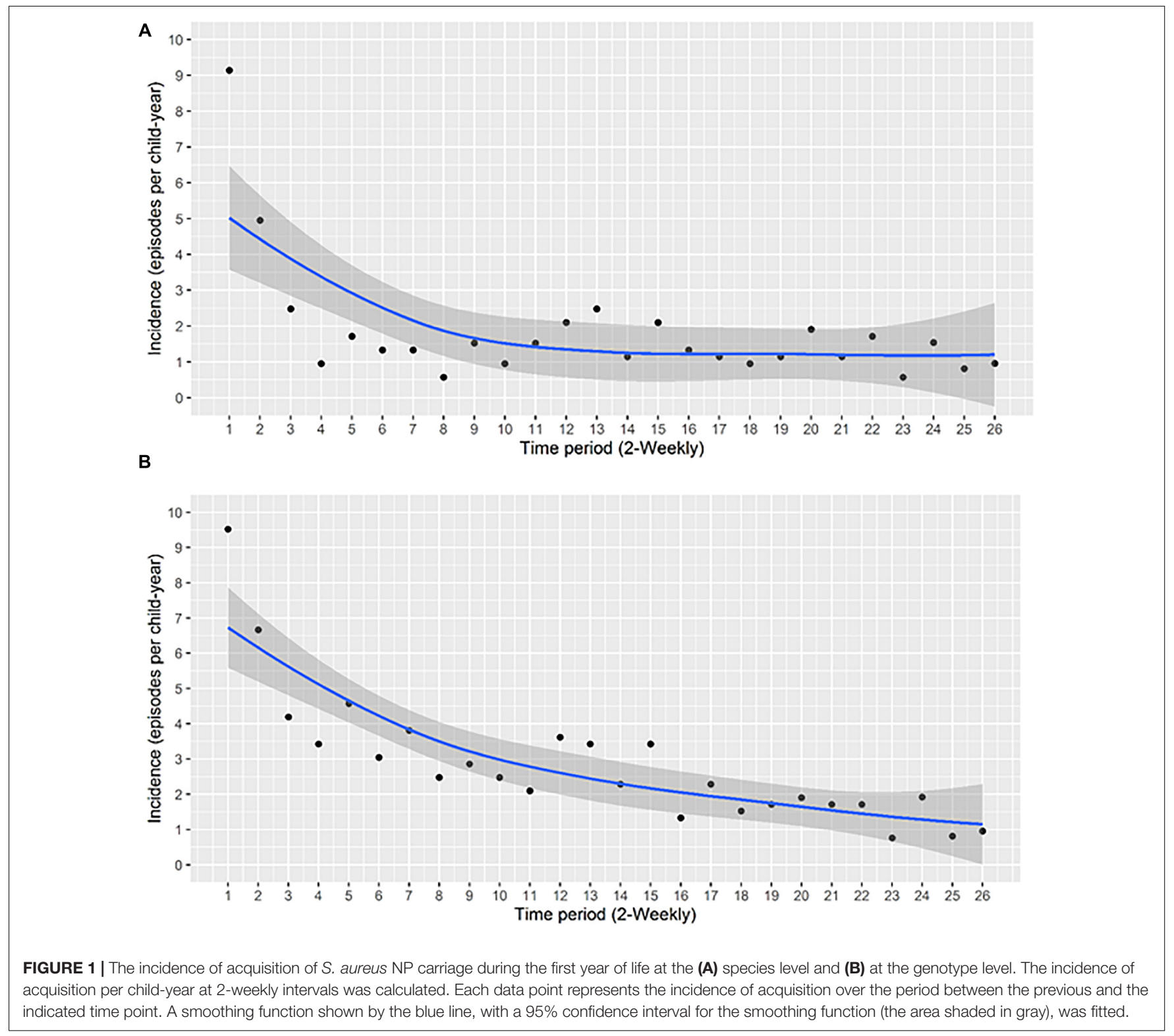


Provincial Child Health Research Committee. Written informed consent was obtained from parents and renewed annually.

\section{Statistical Analysis and Graphical Representation}

An acquisition event was defined according to Miller et al. (2014) as a $S$. aureus-positive culture subsequent to two consecutive negative swab cultures, or the detection of a new spa-CC in a child with previously positive cultures of a different spa-CCs. Loss of $S$. aureus colonization was defined as two consecutive negative swabs for $S$. aureus, or two consecutive swabs without the previous spa-CC (Miller et al., 2014). The acquisition and loss events were estimated to occur at the midpoint between the last negative and the first positive swab, and between the last positive and first negative swab, respectively. The duration of colonization by a genotype (spa-CC) was measured as the time between acquisition and loss. The median permutation test was used to assess the significance in the difference between median carriage duration of individual genotypes. Recurrent acquisition episodes both at the species and the genotype levels were determined using the conditional gap-time model. The Cox proportional hazards model was used to investigate the determinants of time to first $S$. aureus acquisition; variables with $p$-values of $<0.2$ in the univariable models were included in a multivariable model.

The carrier index (the proportion of samples collected from each infant during the first year of life which were positive for S. aureus) was calculated in order to define $S$. aureus carriage patterns: persistent (carrier index $\geq 0.8$ ), prolonged (carrier index $\geq 0.5)$, intermittent $(0<$ carrier index $\leq 0.5)$, and no carriage (carrier index $=0$ ). Two genotype diversity measures were used: the absolute number of genotypes (spa-CCs) carried by each infant, and the Shannon-Weiner Diversity Index, a measure of alpha diversity (the richness and evenness of taxa within a single

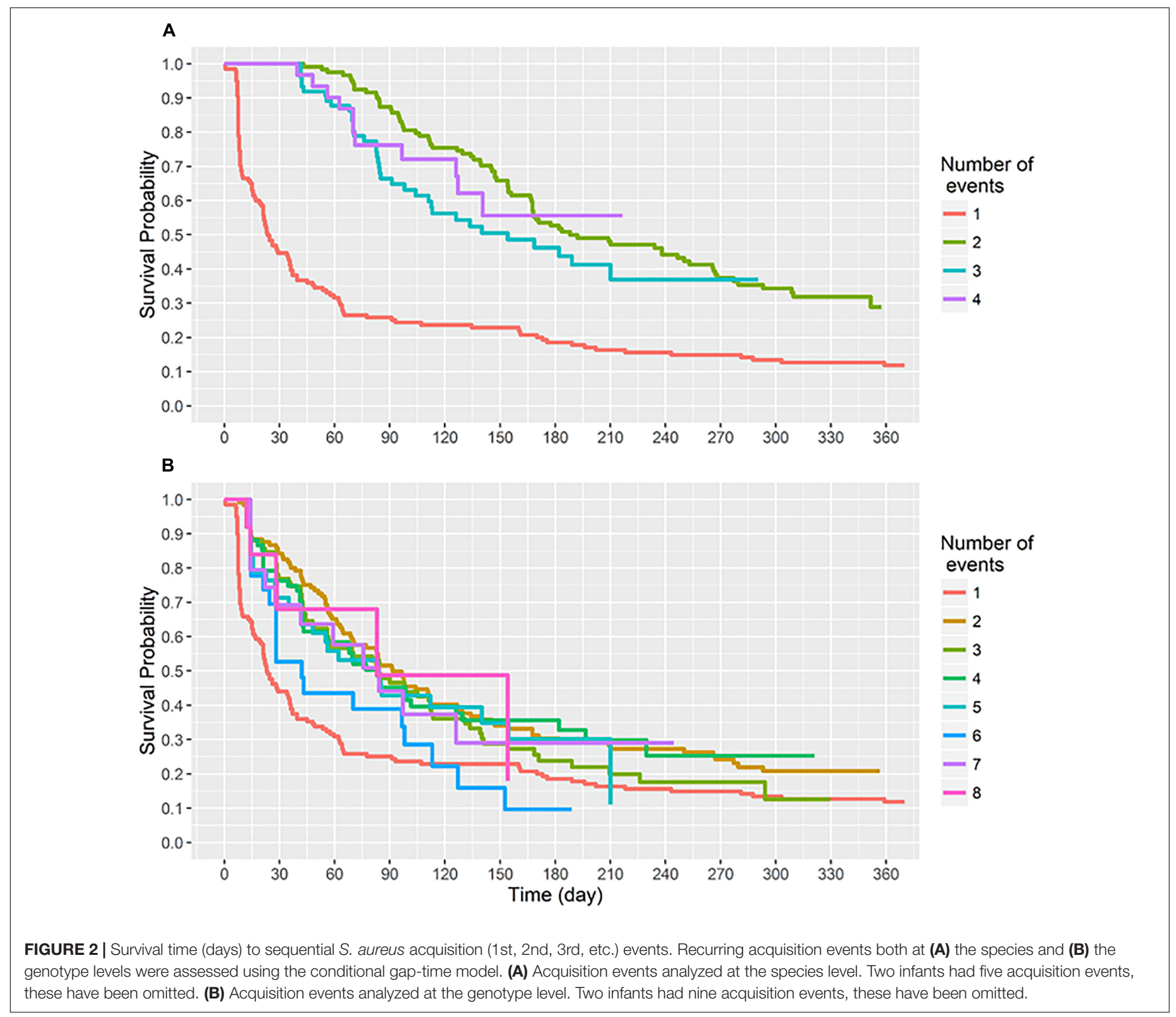


sample) (Shannon, 1948; Simpson, 1949). In this study, the alpha diversity was applied to measure the variation in carriage of different genotypes of each infant over the first year of life. Pearson's correlation coefficient for the carrier index and both of the genotype diversity measures was estimated. Analysis was collectively performed in $\mathrm{R}$ version 3.1.2 ( $\mathrm{R}$ Foundation for Statistical Computing, Vienna, Austria) (R Core Team, 2014).

\section{RESULTS}

The clinical and demographic characteristics of 137 motherinfant pairs are summarized in Table 1. The median gestational age was 39 weeks (Inter-quartile range (IQR) 38-39 weeks); 56\% (77/137) of the infants were female. A total of 3417 infant NP swabs were collected. Swabs were collected 2-weekly (median 14 days; IQR 13-15) from each infant for the first year of life. The median number of NP swabs collected per infant was 24 (IQR; 23-26) with a minimum of 18 swabs. There were 125 maternal NP swabs obtained at the time of birth, of which $21 / 125$ (17\%) yielded S. aureus on culture. S. aureus was isolated from $21 \%(725 / 3417)$ of infant NP swabs. Overall, 88\% (121/137) of infants acquired S. aureus at least once during their first year of life. Of 578 isolates BURP analysis clustered 71 different spa-types into 11 spa-CCs.
Eleven of the $71 s p a$-types represented singletons (116 isolates) and three spa-types (27 isolates) were excluded from the cluster analysis due to the small number of repeat regions.

\section{Incidence of S. aureus Acquisition at the Species and Genotype Levels}

A total of 248 acquisition events occurred during the study period, yielding an overall acquisition incidence of 1.83 episodes per child-year (95\% CI 1.61-2.07). The highest incidence was observed during the first 2 weeks of age, with 9.2 episodes per child-year (95\% CI 6.91-12.08) (Figure 1A).

At the genotype level, the total number of acquisition events was 390 with an overall acquisition incidence of 2.8 events per child-year (95\% CI 2.6-3.1). The highest incidence occurred during the first 2 weeks of life, with 9.5 episodes per childyear (95\% CI 7.2-12.5), thereafter, a gradual decrease was observed (Figure 1B). Overall, we noted higher acquisition incidence rates at the genotype level compared to the species level (Figures 1A,B).

By 91 days of age $75 \%$ of infants had acquired S. aureus for the first time (Figure 2A). First acquisition occurred at a median age of 23 days (95\% CI 21-36 days). Of the 121 carrier infants, $64 \%(n=77)$ acquired $S$. aureus for a second time at median age of 188 days (95\% CI, $168-266$ days); 31\% $(n=37)$ acquired

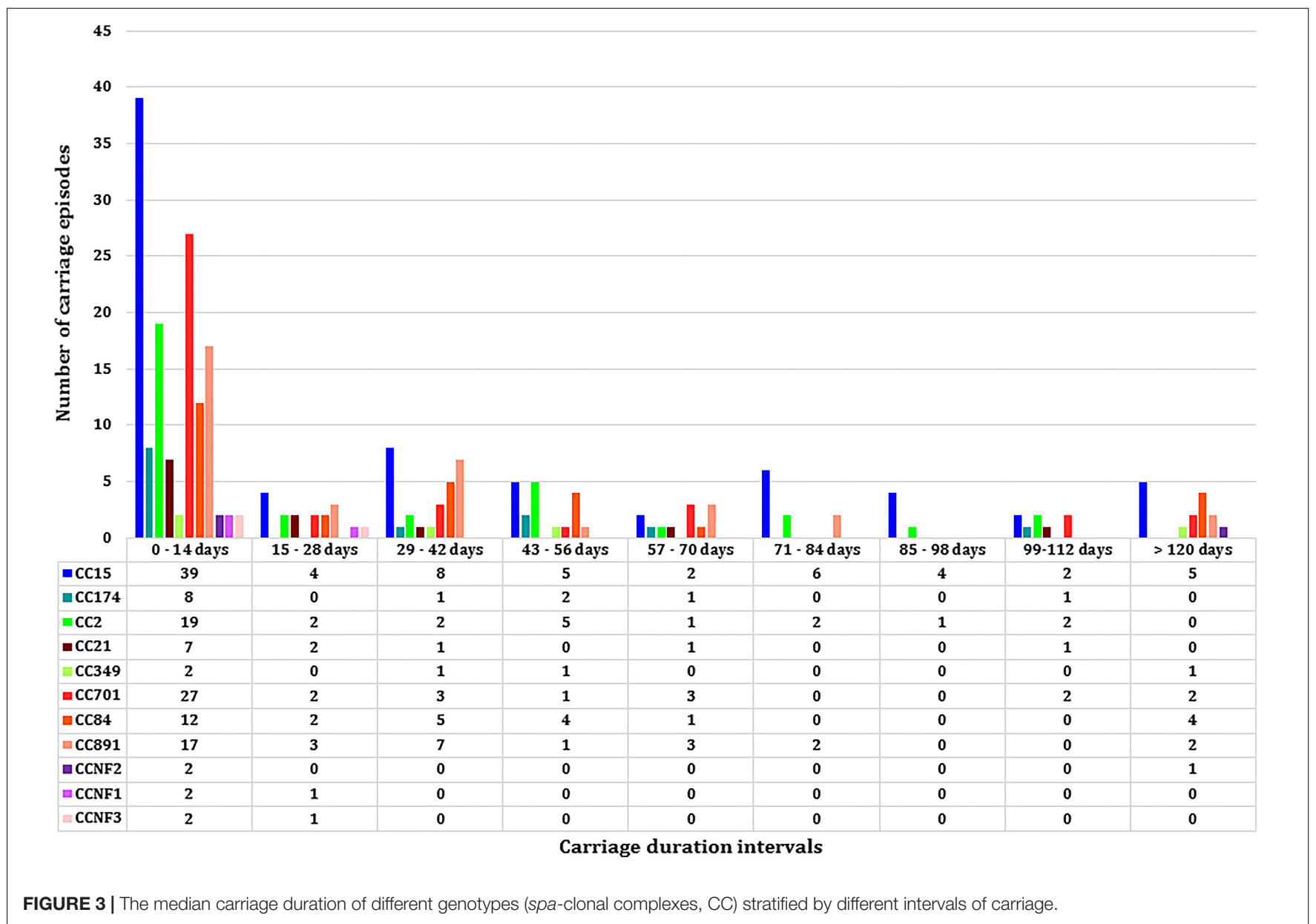


S. aureus a third time, and $9 \%(n=11)$ of carrier infants had four acquisition events during the first year of life (Figure 3). At the genotype level, 75\% (91/121) of the infants who had a first acquisition, had a second acquisition event (either identical, related or a different spa-type) at a median age of 91.5 days (95\% CI, 70.5-126.5 days) (Figure 2B). A total of nine new acquisition events at the genotype level occurred in two infants.

\section{Determinants of First Acquisition of Staphylococcus aureus}

The Cox proportional hazards model was used to determine covariates associated with time to $S$. aureus first acquisition (Table 2). A greater number of individuals ( $\geq 5$ individuals) living in the home [Hazard Ratio (HR) 1.06, 95\% CI 1.07-1.43, $p=0.01$, having a $S$. aureus carrier mother (HR; 1.5, 95\% CI 1.2-2.47, $p=0.03$ ), and infants having a positive TST at any time during the first year of life (HR; 1.81, 95\% CI 0.973.3, $p=0.06$ ) were all associated with earlier first $S$. aureus

TABLE 2 | Univariable and multivariable analysis of factors associated with time to S. aureus first acquisition.

\begin{tabular}{|c|c|c|c|}
\hline \multirow[t]{2}{*}{ Factor } & \multicolumn{3}{|c|}{$\begin{array}{c}\text { Hazard of time to first acquisition of } \\
\text { S. aureus }\end{array}$} \\
\hline & HR & (95\% confidence interval) & $p$-Value \\
\hline \multicolumn{4}{|l|}{ Univariable } \\
\hline HIV-exposure & 0.92 & $0.60-1.39$ & 0.70 \\
\hline Day care attendance & 1.29 & $0.75-2.23$ & 0.84 \\
\hline Preterm & 0.94 & $0.62-1.42$ & 0.78 \\
\hline $\begin{array}{l}\text { Having an animal in the } \\
\text { household }\end{array}$ & 0.97 & $0.66-1.39$ & 0.88 \\
\hline Smoking mother & 0.84 & $0.57-1.23$ & 0.38 \\
\hline Smoking father & 0.82 & $0.56-1.18$ & 0.29 \\
\hline $\begin{array}{l}\text { More than } 5 \text { people in } \\
\text { household }\end{array}$ & 1.06 & $1.01-1.12$ & 0.02 \\
\hline $\begin{array}{l}\text { Delivery mode (Vaginal vs. } \\
\text { cesarean) }\end{array}$ & 0.80 & $0.44-1.47$ & 0.48 \\
\hline Maternal S. aureus carriage & 1.57 & $0.96-2.57$ & 0.06 \\
\hline Infant hospital admission & 0.74 & $0.34-1.63$ & 0.46 \\
\hline $\begin{array}{l}\text { Formula feeding at } 6 \text { months } \\
\text { (vs. breast-feeding) }\end{array}$ & 1.02 & $0.51-2.07$ & 0.95 \\
\hline $\begin{array}{l}\text { Mixed feeding at } 6 \text { months (vs. } \\
\text { breast-feeding) }\end{array}$ & 1.19 & $0.50-2.82$ & 0.69 \\
\hline $\begin{array}{l}\text { TB infection during the first yea } \\
\text { of life* }\end{array}$ & $\operatorname{rat} 1.29$ & $0.75-2.2$ & 0.24 \\
\hline Having younger sibling & 0.75 & $0.49-1.14$ & 0.18 \\
\hline Low-moderate SES & 1.04 & $0.52-2.07$ & 0.92 \\
\hline Moderate-high SES & 2.20 & $0.85-5.67$ & 0.10 \\
\hline \multicolumn{4}{|l|}{ Multivariable } \\
\hline $\begin{array}{l}\text { More than five people in } \\
\text { household }\end{array}$ & 1.06 & $1.07-1.43$ & 0.01 \\
\hline $\begin{array}{l}\text { TB infection during the first year } \\
\text { of life* }\end{array}$ & $\operatorname{ar} 1.81$ & $0.97-3.3$ & 0.06 \\
\hline Maternal S. aureus carriage & 1.5 & $1.2-2.47$ & 0.03 \\
\hline
\end{tabular}

HR, hazard ratio; TB, tuberculosis; SES, socioeconomic status. *As defined by the tuberculin Skin Test reactivity results. acquisition (Table 2). TST reactivity was significant only in the multivariable analysis, with no interaction observed with the number of individuals in the household.

\section{Defining Carriage Patterns Based on Carriage Duration, Carrier Index, and Genotypic Diversity}

Eleven spa-CCs were identified, which were carried by infants for different lengths of time (Figure 3). A median carriage duration of 14 days (the minimum possible estimated duration, given the definitions used) was observed for eight out of the 11 spaCCs: CC2 (IQR; 14-55 days), CC15 (IQR; 14-70 days), CC21 (IQR; 14-34.5 days), CC174 (IQR; 14-45 days), CC701 (IQR; 14-41 days), clonal complex with no-founder (CCNF) 1 (IQR; 14-28 days), CCNF2 (IQR; 14-126 days), and CCNF3 (IQR; 14-25 days). For the remaining four spa-CCs, longer median carriage duration was observed; 20.5 days for CC891 (IQR; 1442 days), 29.5 days for CC84 (IQR; 14-56 days), and 42 days for CC349 (IQR; 14-52). Duration of carriage of spa-CC15, the most frequently detected spa-type, varied between 0 and 215 days. Using the median permutation test, the only significant difference in the carriage duration was observed between CC84 and CC701 (Table 3).

Based on the carrier index, $12 \%(16 / 137)$ of infants were noncarriers, while the rest of infants (121/137) had indices between 0.03 and 0.74 (Figure 4). Using a carrier index of 0.8 as a cutoff to distinguish between persistent and intermittent carriage, we observed no persistent carriers. Therefore, we elected to refer to infants with a carrier index of more than 0.5 as having "prolonged carriage." Accordingly, 6\% (7/121) of carrier infants had prolonged carriage of $S$. aureus at the species level. However, at the genotype level, only $2 \%(2 / 121)$ of infants had prolonged carriage with a single genotype (Figure 4). Overall, 39\% (47/121) of infants carried only a single genotype during the first year, with a carrier index ranging from 0.03 to 0.61 . A positive correlation $(r=0.5 ; 95 \%$ CI $0.35-0.62, p<0.0001)$ was observed between the absolute number of different spa-CC carried by an infant and the carrier index (Figure 5A). The median alpha diversity observed was 1.3 (IQR; 1.03-1.52). A positive correlation was also observed between alpha diversity and carrier index $(r=0.6 ; 95 \%$ CI 0.46-0.70, $p<0.0001$ ) (Figure 5B).

\section{DISCUSSION}

This study is unique in applying genotypic measures of strain diversity to describe the dynamics of $S$. aureus NP colonization in a frequently sampled cohort of African infants during the first year of life. Key findings include a high incidence of $S$. aureus acquisition at the genotype level, the absence of persistent carriage and a correlation between increased carriage frequency and measures of strain diversity. We also noted a high incidence of acquisition at the genotype level within the first 4 weeks of life. There was no clear association between strain genotype and carriage duration. TST reactivity was associated with reduced time to first acquisition of $S$. aureus. 
TABLE 3 | Significance level for pairwise comparison of differences in median carriage duration for the 11 spa-CCs.

\begin{tabular}{|c|c|c|c|c|c|c|c|c|c|c|c|}
\hline & $\mathrm{CC} 2$ & CC15 & CC21 & CC84 & CC174 & cc346 & CC701 & CC891 & CCNF1 & CCNF2 & CCNF3 \\
\hline CC15 & & NA & 0.63 & 0.19 & 0.46 & 0.18 & 0.18 & 0.70 & 0.46 & 0.47 & 0.46 \\
\hline CC84 & & & & NA & 0.47 & 0.65 & 0.03 & 0.54 & 0.25 & 0.43 & 0.30 \\
\hline CC174 & & & & & NA & 0.18 & 0.08 & 0.58 & 0.29 & 0.30 & 0.31 \\
\hline CC346 & & & & & & NA & 0.07 & 0.17 & 0.11 & 0.48 & 0.12 \\
\hline CC891 & & & & & & & & NA & 0.64 & 0.43 & 0.60 \\
\hline CCNF1 & & & & & & & & & NA & 0.39 & 0.40 \\
\hline CCNF2 & & & & & & & & & & NA & 0.40 \\
\hline CCNF3 & & & & & & & & & & & NA \\
\hline
\end{tabular}

CC; clonal complex, NF; no founder, NA; not applicable. Significant difference would be less than 0.05 . The table shows the raw permutation values. No significant differences were observed between the median carriage durations for each of the spa-CCs except for CC701 and CC84 (in bold).

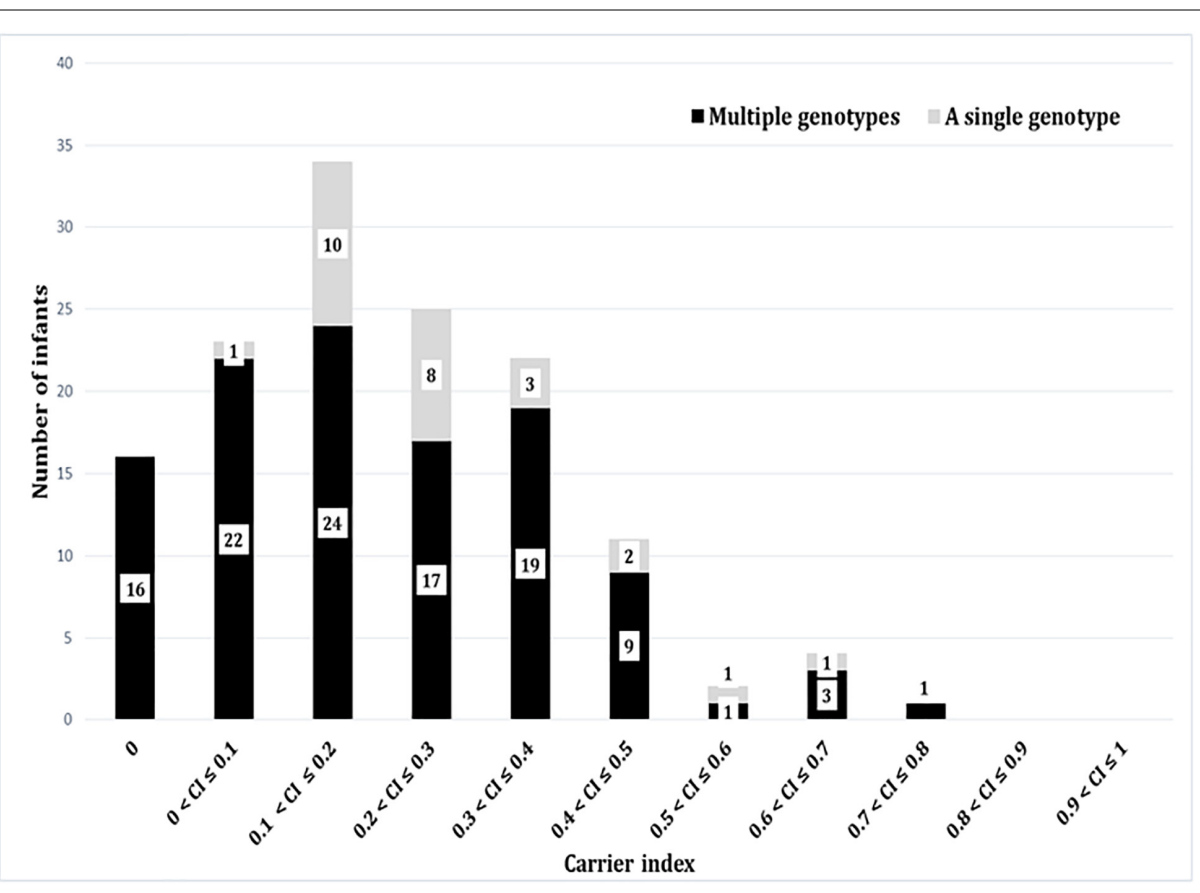

FIGURE 4 | The carrier indices for 137 infants followed up longitudinally every 2 weeks for the first year of life. The carrier index is defined as the number of $S$. aureus positive swabs divided by the total number of NP swabs collected per infant during the study period. The gray bars represent number of infants with specified carrier index who carried a single genotype in all positive swabs, whilst dark bars represent number of infants who carried multiple different genotypes at different time points. For example, 34 infants had a carrier index of greater than 0.1 and less than or equal to 0.2 . Of these 34 infants, 10 infants carried a single genotype over the period of study, whilst 24 carried multiple genotypes.

Staphylococcus aureus carriage in our cohort was less common than that previously described in another African cohort study. By 30 days of age $45 \%$ of infants had acquired S. aureus for the first time, compared with $75 \%$ at the same age in The Gambia (Kwambana et al., 2011). A possible reason for the higher carriage rates among infants from The Gambia may be climate, as The Gambia has considerably higher annual temperatures. In a review, Leekha et al. (2012) report a trend toward increased $S$. aureus carriage and infections during the hot season.

Large family size ( $>5$ individuals), and $S$. aureus maternal carriage were associated with reduced time to $S$. aureus first acquisition; these findings are in line with previous studies (Bourgeois-Nicolaos et al., 2010; Lebon et al., 2010; Soltani et al., 2014). Previous studies have used TST (induration reaction of $>10 \mathrm{~mm}$ ) as a measure of $\mathrm{TB}$ infection in settings where the Bacillus Calmette-Guerin (BCG) vaccine is administered (Martinez et al., 2018; Pelzer et al., 2018). We report an association between pediatric TB infection (as defined by TST) and a shorter time to first acquisition of S. aureus, possibly reflecting unmeasured exposures to both organisms, for example, exposure to health care facilities or antimicrobial therapy. This observation requires further 

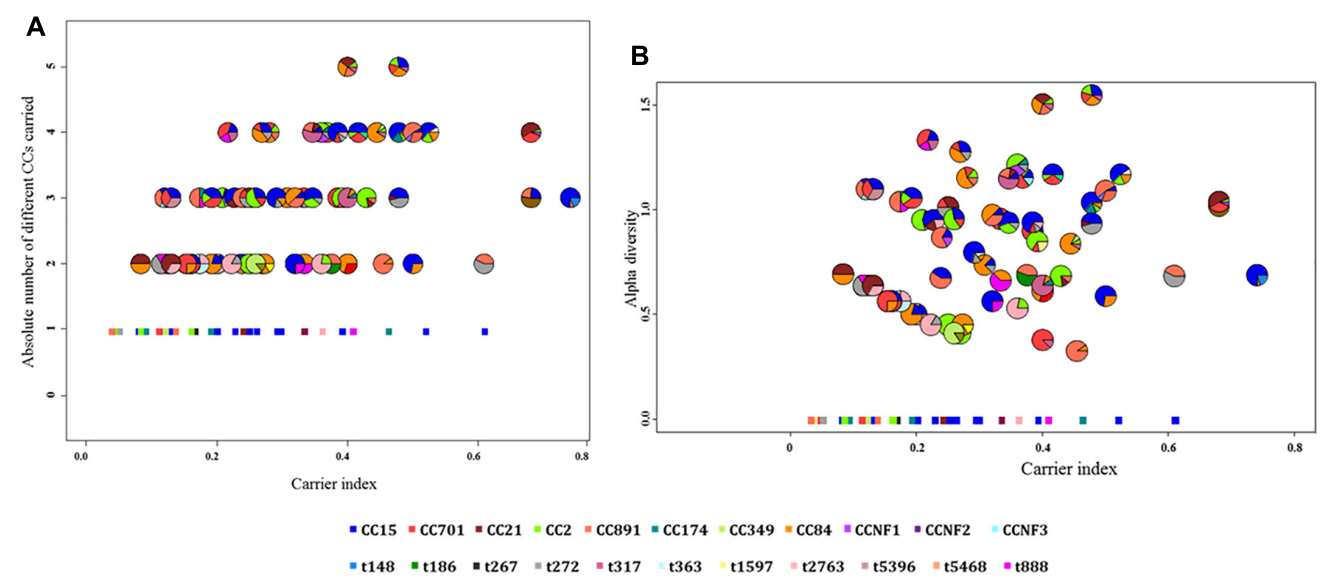

FIGURE 5 | The correlation between the carrier index and (A) absolute number of spa-CC or (B) alpha diversity. (A) The carrier index vs. absolute number of spa-CC carried by each infant showing a positive correlation $(r=0.5, p<0.0001)$. (B) The carrier index vs. alpha diversity showing a stronger positive correlation $(r=0.6$, $p<0.0001)$. The carrier index was calculated as the proportion of $S$. aureus positive samples divided by the total number of samples collected for each infant. The alpha diversity as measured by the $\mathrm{H}$-index reflects the number and distribution of genotypes carried by each infant. The minimum value for the absolute number of spa-CC is 1, while the minimum alpha diversity is zero. Each pie chart represents the proportions of different genotypes carried by a single infant throughout the first year. The square dots in both figures indicate infants who carried only a single genotype in all positive samples.

investigation. Previous studies have shown that cigarette smoke exposure was negatively associated with $S$. aureus NP carriage, however, we did not observe any association between either maternal of paternal smoking and $S$. aureus first acquisition in infants (Chatzakis et al., 2011).

The observed high incidence rate early in life could not be explained by the large family size, maternal $S$. aureus carriage or TB infection. However, in previous studies, S. aureus carriage among healthy infants was shown to have a negative association with age (i.e., carriage decrease with increasing age). This could be explained by the microbial interference within the nasopharynx, where $S$. aureus is the predominant colonizer for the first 6 weeks namely and it gets replaced mainly by the Streptococcus pneumonia, Haemophilus influenzae, and Moraxella catarrhalis with increasing age (Biesbroek et al., 2014).

Twelve percent of infants in our study were non-carriers. None of the infants had a carrier index of 0.8 or more (previously used to define persistent carriage), at either the species or genotype level. This is in agreement with longitudinal studies from the United Kingdom and the Netherlands where persistent carriage was rarely found during infancy (Peacock et al., 2003; Lebon et al., 2008). However, in a study from Belgium which included 333 preschool children aged 3-6 years, $15 \%$ of the children were classified as persistent carriers based on consecutive carriage of the same genotype (Blumental et al., 2013).

A novel finding was that when combining the carrier index with measures of genotypic diversity, a higher carrier index was associated with increased genotypic diversity, i.e., infants with more frequent or prolonged carriage also harbored more genotype diversity. This observation is in contrast with adult studies where persistent carriers were observed to often carry the same genotype for prolonged periods (Hu et al., 1995; Van Belkum et al., 1997). For example, one study of 104 healthy adults, in which a median number of 14 swabs was collected over 19 months, showed that $14 \%$ of participants carried a single genotype with a carrier index of 0.9 or higher (Eriksen et al., 1995). In another study which involved 91 university staff and was conducted over 12 weeks, $36 \%$ had carrier indices of 0.8 or higher. Participants with carrier index of 1.0 were found to carry the same strain 8 years later (Vandenbergh et al., 1999). The NP microbiota in early life is highly unstable, and is repeatedly disturbed by intercurrent viral infections (van den Bergh et al., 2012; Biesbroek et al., 2014). This changing ecological environment may influence the ability of $S$. aureus strains to colonize the NP persistently, which might explain the high genotype diversity amongst infants, in contrast to adult studies.

The importance of including genotypic measures of strain diversity is demonstrated by our study. For example, seven infants carried $S$. aureus with a carrier index of greater than 0.5 , but only two of them carried a single strain throughout the first year. There are few studies which have longitudinally investigated NP carriage of $S$. aureus at the genotype level among healthy infants (Peacock et al., 2003; Lebon et al., 2008). In a study of 100 healthy infants from the United Kingdom with nine NP swabs per infant over the first 6 months of life, $25 \%$ of infants had no detectable NP carriage, and most infants carried a single genotype regardless of whether the carriage pattern was persistent or intermittent (Peacock et al., 2003). In a birth cohort study in the Netherlands, three NP swabs were collected from 443 infants over 14 months, with only two swabs collected during the first year of life (at 1.5 and 6 months). All three swabs were negative for $S$. aureus in $36 \%$ of infants, while, only two infants carried a single strain in all three swabs (Lebon et al., 2008). Verhoeven et al. (2012) suggested that a total of seven swabs collected over 5 weeks are sufficient to distinguish persistent carriage from the 
other patterns. However, non-carriage and persistent carriage rates tend to decrease with increasing follow-up periods and decreasing culture intervals (Vandenbergh et al., 1999). In our study, a median of $24 \mathrm{NP}$ swabs were collected per infant over 1 year, with a minimum of 18 swabs, compared to three and nine in the studies from the Netherlands and the United Kingdom, respectively. The intense 2 -weekly sampling is a key and unique feature of this study; our data may provide a more accurate estimate of "true" non-carriage (in our study estimated at 12\%) and persistent/prolonged carriage.

The carriage duration as well as the diversity of the genotypes carried by an individual are among the criteria used in previous studies to distinguish intermittent from persistent carriers; persistent $S$. aureus carriers tend to carry fewer strains for longer periods of time (Van Belkum et al., 2009; Blumental et al., 2013; Muthukrishnan et al., 2013). In this study, neither the carriage duration nor genotype diversity was able to segregate the infants into distinct groups. In fact, infants with prolonged carriage harbored more diverse genotypes. Although spa-CC15 was previously found to be less likely associated with long term compared to intermittent carriage (Miller et al., 2014), in this study no association between carriage duration and genotype was observed.

Limitations of our study include that we did not specifically look for co-carriage with multiple strains in the same individual, and infants were studied for a only 1 year. However, this study represents one of the most intensely sampled cohorts reported. Extended follow up is currently underway to identify when children first develop true prolonged carriage with the same strain, as has been observed in adults. Strengths of our study include the longitudinal nature of the study, allied to the comprehensive epidemiological data collection and strain typing, which allowed for detailed analysis of colonization dynamics at the strain level. The approach of combining genotype diversity and carrier index may be useful for future studies describing the epidemiology of colonization with S. aureus.

\section{CONCLUSION}

In conclusion, we genotyped $S$. aureus isolates from the NP of intensively sampled infants from South Africa to extend

\section{REFERENCES}

Abdulgader, S. (2016). Nasopharyngeal Carriage With Staphylococcus Aureus in Healthy Children During the First Year of Life - The Drakenstein Child Health study. Ph.D. thesis, University of Cape Town, Cape Town.

Armstrong-Esther, C. A., and Smith, J. E. (1976). Carriage patterns of Staphylococcus aureus in healthy non-hospital population of adults and children. Ann. Hum. Biol. 3, 221-227. doi: 10.1080/03014467600 001381

Biesbroek, G., Tsivtsivadze, E., Sanders, E. A. M., Montijn, R., Veenhoven, R. H., Keijser, B. J. F., et al. (2014). Early respiratory microbiota composition determines bacterial succession patterns and respiratory health in children. Am. J. Respir. Crit. Care Med. 190, 1283-1292. doi: 10.1164/rccm.201407-1240OC

Blumental, S., Deplano, A., Jourdain, S., De Mendonça, R., Hallin, M., Nonhoff, C., et al. (2013). Dynamic pattern and genotypic diversity of Staphylococcus our understanding of carriage dynamics in this age group. Incorporating genotypic measures allowed us to obtain a more accurate assessment of persistent or prolonged carriage. Persistent carriage was not identified, while prolonged carriage, defined by a carrier index of $>0.5$, was uncommon. S. aureus NP carriage is highly dynamic in infants. Increased carrier index was associated with genotype diversity, and there was no clear association between particular genotypes and duration of carriage.

\section{AUTHOR CONTRIBUTIONS}

HZ was the principal investigator of the Drakenstein Child Health Study and conceived and designed the birth cohort study together with MN. SA and MN conceptualized the S. aureus study. LR contributed to the analysis and interpretation of results and development of the manuscript. JR, PN, SG-L, and FD assisted with the statistical analysis and graphical representation. SA drafted this manuscript. All authors reviewed and improved the final version of the manuscript.

\section{FUNDING}

SA was funded by the Organization for Women in Science (OWSD). This work was funded by grants from the Bill and Melinda Gates Foundation Global Health Grant (OPP1017641), and by the Office of the Director, National Institutes of Health (OD), the National Institute of Environmental Health Sciences (NIEHS), and the National Human Genome Research Institute (Grant Numbers 1U01HG006961-01 and 1U54HG009824-01).

\section{ACKNOWLEDGMENTS}

The content is solely the responsibility of the authors and does not necessarily represent the official views of the sponsors. This work has been conducted as part of the first author's Ph.D. degree, and it partially overlaps with the thesis which could be accessed online (Abdulgader, 2016).

aureus nasopharyngeal carriage in healthy pre-school children. J. Antimicrob. Chemother. 68, 1517-1523. doi: 10.1093/jac/dkt080

Bourgeois-Nicolaos, N., Lucet, J. C., Daubié, C., Benchaba, F., Rajguru, M., Ruimy, R., et al. (2010). Maternal vaginal colonisation by Staphylococcus aureus and newborn acquisition at delivery. Paediatr. Perinat. Epidemiol. 24, 488-491. doi: 10.1111/j.1365-3016.2010.01139.x

Chatzakis, E., Scoulica, E., Papageorgiou, N., Maraki, S., Samonis, G., and Galanakis, E. (2011). Infant colonization by Staphylococcus aureus: role of maternal carriage. Eur. J. Clin. Microbiol. Infect. Dis. 30, 1111-1117. doi: 10. 1007/s10096-011-1199-9

Cunliffe, A. C. (1949). Incidence of Staphylococcus aureus in anterior nares of healthy children. Lancet 2, 411-414. doi: 10.1016/S0140-6736(49)90118-X

Eriksen, N. H., Espersen, F., Rosdahl, V. T., and Jensen, K. (1995). Carriage of Staphylococcus aureus among 104 healthy persons during a 19-month period. Epidemiol. Infect. 115, 51-60. doi: 10.1017/S0950268800058118 
Hu, L., Umeda, A., Kondo, S., and Amako, K. (1995). Typing of Staphylococcus aureus colonising human nasal carriers by pulsed-field gel electrophoresis. J. Med. Microbiol. 42, 127-132. doi: 10.1099/00222615-42-2-127

Johannessen, M., Sollid, J. E., and Hanssen, A. (2012). Host and microbe determinants that may influence the success of S. aureus colonization. Front. Cell. Infect. Microbiol. 2:56. doi: 10.3389/fcimb.2012.00056

Kateete, D. P., Kimani, C. N., Katabazi, F. A., Okeng, A., Okee, M. S., Nanteza, A., et al. (2010). Identification of Staphylococcus aureus: DNase and Mannitol salt agar improve the efficiency of the tube coagulase test. Ann. Clin. Microbiol. Antimicrob. 9, 1-7. doi: 10.1186/1476-0711-9-23

Kwambana, B. A., Barer, M. R., Bottomley, C., Adegbola, R. A., and Antonio, M. (2011). Early acquisition and high nasopharyngeal co-colonisation by Streptococcus pneumoniae and three respiratory pathogens amongst Gambian new-borns and infants. BMC Infect. Dis. 11:175. doi: 10.1186/1471-233411-175

Lebon, A., Labout, J. A. M., Verbrugh, H. A., Jaddoe, V. W. V., Hofman, A., van Wamel, W., et al. (2008). Dynamics and determinants of Staphylococcus aureus carriage in infancy: the generation R study. J. Clin. Microbiol. 46, 3517-3521. doi: 10.1128/JCM.00641-08

Lebon, A., Moll, H. A., Tavakol, M., van Wamel, W. J., Jaddoe, V. W. V., Hofman, A., et al. (2010). Correlation of bacterial colonization status between mother and child: the generation R study. J. Clin. Microbiol. 48, 960-962. doi: 10.1128/JCM.01799-09

Leekha, S., Diekema, D. J., and Perencevich, E. N. (2012). Seasonality of staphylococcal infections. Clin. Microbiol. Infect. 18, 927-933. doi: 10.1111/j. 1469-0691.2012.03955.x

Martinez, L., Le Roux, D. M., Barnett, W., Stadler, A., Nicol, M. P., and Zar, H. J. (2018). Tuberculin skin test conversion and primary progressive tuberculosis disease in the first 5 years of life: a birth cohort study from Cape Town, South Africa. Lancet Child Adolesc. Health 2, 46-55. doi: 10.1016/S23524642(17)30149-9

Miller, R. R., Walker, A. S., Godwin, H., Fung, R., Votintseva, A., Bowden, R., et al. (2014). Dynamics of acquisition and loss of carriage of Staphylococcus aureus strains in the community: the effect of clonal complex. J. Infect. 68, 426-439. doi: 10.1016/j.jinf.2013.12.013

Muthukrishnan, G., Lamers, R. P., Ellis, A., Paramanandam, V., Persaud, A. B., Tafur, S., et al. (2013). Longitudinal genetic analyses of Staphylococcus aureus nasal carriage dynamics in a diverse population. BMC Infect. Dis. 13:221. doi: 10.1186/1471-2334-13-221

Noble, W. C., Valkenburg, H. A., and Wolters, C. H. (1967). Carriage of Staphylococcus aureus in random samples of a normal population. J. Hygine 65, 567-573. doi: 10.1017/S002217240004609X

Nouwen, J. L., Ott, A., Kluytmans-Vandenbergh, M. F. Q., Boelens, H. M., Hofman, A., van Belkum, A., et al. (2004). Predicting the Staphylococcus aureus nasal carrier state: derivation and validation of a "culture rule". Clin. Infect. Dis. 39, 806-811. doi: 10.1086/423376

Peacock, S. J., Justice, A., Griffiths, D., de Silva, G. D. I., Kantzanou, M. N., Crook, D., et al. (2003). Determinants of acquisition and carriage of Staphylococcus aureus in infancy. J. Cin. Microbiol. 41, 5718-5725. doi: 10.1128/ JCM.41.12.5718

Pelzer, P. T., Mutayoba, B., and Cobelens, F. G. J. (2018). BCG vaccination protects against infection with Mycobacterium tuberculosis ascertained by tuberculin skin testing. J. Infect. 77, 335-340. doi: 10.1016/j.jinf.2018.03.010

Pohl, K., Francois, P., Stenz, L., Schlink, F., Geiger, T., Herbert, S., et al. (2009). CodY in Staphylococcus aureus: a regulatory link between metabolism and virulence gene expression. J. Bacteriol. 191, 2953-2963. doi: 10.1128/JB. 01492-08

R Core Team (2014). R: A Language and Environment for Statistical Computing. Vienna: R Foundation for Statistical Computing.

Sakr, A., Brégeon, F., Mège, J., Rolain, J., and Gobert, A. P. (2018). Staphylococcus aureus nasal colonization: an update on mechanisms, epidemiology, risk factors, and subsequent infections. Front. Microbiol. 9:2419. doi: 10.3389/fmicb. 2018.02419

Schaumburg, F., Alabi, A. S., Kaba, H., Zoleko, R. M., Diop, D. A., Mackanga, J., et al. (2013). Transmission of Staphylococcus aureus between mothers and infants in an African setting. Epidemiology 20, O390-O396. doi: 10.1111/14690691.12417

Shannon, C. E. (1948). A mathematical theory of communication. Bell Syst. Techn. J. 27, 379-423. doi: 10.1145/584091.584093

Simpson, E. (1949). Measurement of diversity. Nature 163:688. doi: 10.1038/ $163688 \mathrm{a} 0$

Soltani, B., Taghavi Ardakani, A., Moravveji, A., Erami, M., Haji Rezaei, M., Moniri, R., et al. (2014). Risk factors for methicillin resistant Staphylococcus aureus nasal colonization of healthy children. Jundishapur J. Microbiol. 7, 1-6. doi: 10.5812/jjm.20025

Van Belkum, A., Eriksen, N. H., Sijmons, M., van Leeuwen, W., van Den Bergh, M., Kluytmans, J., et al. (1997). Coagulase and protein A polymorphisms do not contribute to persistence of nasal colonisation by Staphylococcus aureus. J. Med. Microbiol. 46, 222-232. doi: 10.1099/00222615-463-222

Van Belkum, A., Verkaik, N. J., De Vogel, C. P., Boelens, H. A., Verveer, J., Nouwen, J. L., et al. (2009). Reclassification of Staphylococcus aureus nasal carriage types. J. Infect. Dis. 199, 1820-1826. doi: 10.1086/599119

van den Bergh, M. R., Biesbroek, G., Rossen, J. W., de Steenhuijsen Piters, W. A., Bosch, A. A., van Gils, E. J., et al. (2012). Associations between pathogens in the upper respiratory tract of young children: interplay between viruses and bacteria. PLoS One 7:e47711. doi: 10.1371/journal.pone.0047711

Vandenbergh, M., Yzerman, E. P., and Van Belkum, A. (1999). Follow-up of Staphylococcus aureus nasal carriage after 8 years: redefining the persistent carrier state. J. Cin. Microbiol. 37, 3133-3140.

Verhoeven, P. O., Gagnaire, J., Botelho-Nevers, E., Grattard, F., Carricajo, A., Lucht, F., et al. (2014). Detection and clinical relevance of Staphylococcus aureus nasal carriage: an update. Expert. Rev. Anti Infect. Ther. 12, 75-89. doi: 10.1586/ 14787210.2014.859985

Verhoeven, P. O., Grattard, F., Carricajo, A., Lucht, F., Cazorla, C., Garraud, O., et al. (2012). An algorithm based on one or two nasal samples is accurate to identify persistent nasal carriers of Staphylococcus aureus. Clin. Microbiol. Infect. 18, 551-557. doi: 10.1111/j.1469-0691.2011. 03611.x

Waters, N. R., Samuels, D. J., Behera, R. K., Livny, J., Rhee, K. Y., Sadykov, M. R., et al. (2016). A spectrum of CodY activities drives metabolic reorganization and virulence gene expression in Staphylococcus aureus. Mol. Microbiol. 101, 495-514. doi: 10.1111/mmi.13404

Wertheim, H. F., Melles, D. C., Vos, M. C., van Leeuwen, W., van Belkum, A., Verbrugh, H. A., et al. (2005). The role of nasal carriage in Staphylococcus aureus infections. Lancet Infect. Dis. 5, 751-762. doi: 10.1016/S1473-3099(05) 70295-4

Zar, H. J., Barnett, W., Myer, L., Stein, D. J., and Nicol, M. P. (2014). Investigating the early-life determinants of illness in Africa: the drakenstein child health study. Thorax 70, 592-594. doi: 10.1136/thoraxjnl-2014-206242

Conflict of Interest Statement: The authors declare that the research was conducted in the absence of any commercial or financial relationships that could be construed as a potential conflict of interest.

Copyright $\odot 2019$ Abdulgader, Robberts, Ramjith, Nduru, Dube, Gardner-Lubbe, Zar and Nicol. This is an open-access article distributed under the terms of the Creative Commons Attribution License (CC BY). The use, distribution or reproduction in other forums is permitted, provided the original author(s) and the copyright owner(s) are credited and that the original publication in this journal is cited, in accordance with accepted academic practice. No use, distribution or reproduction is permitted which does not comply with these terms. 